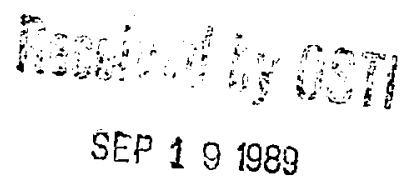

BNL -43118

DE $90 \quad 001088$

\title{
INVESTIGATION OF THE INTERMEDIATE-ENERGY DEUTERON BREAKUP REACTION*
}

\author{
M. Divadeenam and T.E. Ward \\ Neutral Beam Division, Brookhaven National Laboratory, Upton, NY 1973
}

\author{
M.G. Mustafa \\ Lawrence Livermore National Laboratory, Livermore, CA 94550 \\ T. Udagawa and T. Tamuraf \\ University of Texas, Austin, TX 78712
}

\begin{abstract}
The Udagawa-Tamura formalism is employed to calculate the proton singles both in the bound and unbound regions. Both the Elastic-Breakup (EB) and the Breakup-Fusion (BF) processes are considered to calculate the doubly-differential cross section for light and intermediate mass nuclei. The calculated spectra for 25 and $56 \mathrm{MeV}$ deuterons reproduce the experimental spectra very well except for the spectra at large angle and at low energies, of the outgoing particle. Contributions due to precompound and evaporation processes are estimated to supplement the spectral results based on the Elastic-Breakup and BreakupFusion mechanisms. An extension of the model calculations to higher deuteron energies is being made to test the $(\mathrm{EB}+\mathrm{BF})$ model limitations.
\end{abstract}

\section{INTRODUCTION}

Intermediate energy nucleon induced neutron and proton spectra have been successfully interpreted in terms of the Intranuclear Cascade model and the evaporation model. Similarly the precompound and evaporation models have been successful in interpreting the nucleon spectra measured in the nucleon induced and heavy ion induced reactions. However, the application of the models to light ions especially to deuteron induced nucleon spectra has had limitations due to the breakup of the projectile within the nuclear *Work performed under the auspices of the U.S. Department of Energy under contract no. DE-AC02-76CH00016 DISCLAIMER

†Deceased
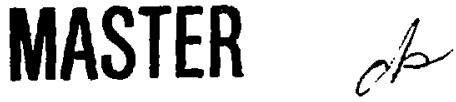

OASTRIBUTION OF THIS DOCUMENT IS UMLIMITEU
This report was prepared as an account of work sponsored by an agency of the United States Government. Neither the United States Government nor any agency thereof, nor any of their employees, makes any warranty, express or implied, or assumes any legal liability or responsibility for the accuracy, completeness, or usefulness of any information, apparatus, product, or process disclosed, or represents that its use would not infringe privately owned rights. Reference herein to any specific commercial product, process, or service by trade name, trademark, manufacturer, or oherwise does not necessarily constilute or imply its endorsement, recommendation, or favoring by the United States Government or any agency thereof. The views and opinions of authors expreseod herein do not necesserily state or reflect those of the United States Government or any agency thereor. 
and coulomb fields. The nuclear reaction model predictability of the deuteron induced neutron and proton spectra at intermediate projectile energy is highly desirable in the application area. The study of deuteron breakup reaction is being extended to intermediate energies following a successful interpretation of the medium energy data.

We aim to develop systematics of deuteron induced nucleon spectra over a wide range of projectile energy and target mass for use in the application area. Towards achieving such a goal we have undertaken the study of deuteron breakup mechanism. The breakup aspects of the deuteron induced reaction have been studied by Udagawa and Tamura ${ }^{1}$, (UT) and Baver et $\mathrm{al}^{2}$. We make use of the UT formalism to calculate the proton singles spectra both in the bound and unbound regions. Contributions due to precompound and evaporation process are estimated to supplement the predictions based on breakup mechanism. Preliminary results of our study were presented at the APS/DNP Santa-Fe meeting ${ }^{3}$.

\section{REACTION MECHANISMS}

Depending on the energy of the projectile, different reaction mechanisms have to be considered in fully describing the reaction process. In the case of deuteron at 25 to 200 $\mathrm{MeV}$ and beyond the following mechanism may be relevant:

1. Breakup-Fusion

2. Evaporation

3. Precompound

1. Breakup-Fusion:

Over the past few years Udagawa and Tamura have extended their MSDR approach to include light/heavy ion breakup-fusion mechanism for calculating both the bound region and continuum spectra of outgoing particles.

The breakup-Fusion of the deuteron reaction may be symbolically written as:

$$
d+A \rightarrow p+n+A \quad[\text { Elastic Breakup (EB)] }
$$




$$
p+n+A \rightarrow p+B \quad[\text { Breakup-Fusion (BF)] }
$$

The sum of partial cross sections for EB and BF processes is:

$$
\frac{d^{2} \sigma^{D R}}{d E_{p} d \Omega_{p}}=\sum_{\ell_{n} j_{n}}=\frac{d^{2} \sigma_{\ell_{n} j_{n}}^{D R}}{d E_{p} d \Omega_{p}}=\sum_{\ell_{n} j_{n}} \frac{d^{2} \sigma_{\ell_{n} j_{n}}^{E B}}{d E_{p} d \Omega_{p}}+\sum_{\ell_{n} j_{n}} \frac{d^{2} \sigma_{\ell_{n} j_{n}}^{B F}}{d E_{p} d \Omega_{p}}
$$

See ref. 1 for details regarding the evaluation of the EB and BF cross sections.

The Elastic Breakup process is relevant only for the outgoing proton energy above zero $\left(E_{p}>0\right)$, where as the Breakup Fusion cross section must be evaluated both for positive and negative values of $\mathrm{E}_{p}$. For $\mathrm{E}_{p}<0$ the Breakup-Fusion cross section is just the stripping (DWBA) cross section to the discrete states in the bound region.

2. Evaporation and Precompound:

For a proper description of the particle spectra in the low and intermediate energy range both the evaporation and precompound mechanisms have to be considered in addition to the direct reaction process. In the present study we make use of Weisskopf-Ewing evaporation theory along with Blann's Geometry dependent hybrid model.4

\section{MODEL CALCULATIONS}

For the purpose of comparing the theoretical calculated doubly-differential cross sections with the relevant experimental data, the total reaction cross section is decomposed as:

$$
\sigma_{R}=\sigma^{D R}+\left[\sigma^{P E}+\sigma^{C N}\right]=\left[\sigma_{d p}+\sigma_{d n}+\sigma_{d d^{1}}+\cdots\right]+\left[\sigma^{P E}+\sigma^{C N}\right]
$$

In the present study we calculate $\sigma_{R}, \sigma_{d p}, \sigma^{P E}$ and $\sigma^{C N}$ and make some assumptions regarding $\sigma_{d n}\left(\approx \sigma_{d p}\right)$ and $\sigma_{d d^{\prime}}$ (a few percent of $\left.\dot{\sigma}_{R}\right)$ and normalize the sum $\left(\sigma^{P E}+\sigma^{C N}\right.$ ) to $\left(\sigma_{R}-\sigma^{D R}\right)$ before adding the corresponding contribution to the UT Breakup-Fusion theory calculated doubly-differential cross sections. 
Blann's OVERLAID ALICE code ${ }^{4}$ was used to estimate the precumpound and evaporation contribution to the total reaction cross section for $(d, p)$ spectra. No parameter variation was entertained in exciton and evaporation model calculations with ALICE code. The ALICE code predicted cross sections were normalized to account for the $\sigma^{D R}$ part of total reaction cross section.

We present a comparison of the Breakup-Fusion plus Exciton and Evaporation model calculated results for the following cases:

1. ${ }^{62} \mathrm{Ni}(d, p), E_{d}=25.5 \mathrm{MeV}$

2. ${ }^{27} \mathrm{Al}(d, p),{ }^{58} \mathrm{Ni}(d, p), E_{d}=56 \mathrm{MeV}$

Figure 1 taken from ULT's paper ${ }^{1}$ presents a comparison of ${ }^{62} \mathrm{Ni}(d, p)$ experimental and calculated spectra for $E_{d}=25.5 \mathrm{MeV}$ and $\theta_{p}=20^{\circ}$. The smooth curve generated by ULT required a normalization factor of $\mathbf{1 . 2}$ to fit the experimental data, however the lowenergy data were not reproduced well. The dashed curve which includes the contribution due to precornpound and evaporation processes does fit the data well without requiring any normalization factor.

For the ${ }^{58} \mathrm{Ni}(\mathrm{d}, \mathrm{p})$ spectra at $\mathrm{E}_{d}=56 \mathrm{MeV}$ and $\Theta=9.5^{\circ}$ and $\theta=30^{\circ}$ the sum of EB and BF contribution is compared with the corresponding experimental data in figure 2a. Figure $2 \mathrm{~b}$ presents a similar comparison for ${ }^{27} \mathrm{Al}(\mathrm{d}, \mathrm{p})$ forward angle spectra. The agreement between theory and experiment is quite satisfactory.

At $E_{d}=56 \mathrm{MeV}$ the evaporation contribution is negligible and precompound emission contribution is also not as important in the quasi-elastic bump region as it is at a lower deuteron energy.

\section{SUMMARY AND FUTURE IMPROVEMENTS}

Based on the test cases studied, the UT Breakup-Fusion reaction formalism for deuteron breakup supplemented with the precompound and evaporation mechanisms does reproduce the deuteron induced proton double differential spectra for $E_{d} \leq 56 \mathrm{MeV}$. In the present study only the low angle data have been investigated. Only recently some 
intermediate-energy deuteron induced neutron spectra both at forward and backward angles have become available. We are extending the model calculations to higher deuteron energies and to heavier targets.

The inelastic breakup and Coulomb effects become important with increasing deuteron energy and target mass and, we plan to pursue these aspects of deuteron breakup mechanism. In addition the ambiguity of the optical model potential will be minimized by making use of local equivalent potentials derived by Mishra et $\mathrm{al}^{5}$. 


\section{FIGURE CAPTIONS}

Fig. 1a. ${ }^{62} \mathrm{Ni}(\mathrm{d}, \mathrm{p}), \mathrm{E}_{d}=25.5 \mathrm{MeV}$; proton spectra at $\Theta_{p}=20^{\circ}$. The solid and dashed curves correspond to Breakup-Fusion calculated results without and with the inclusion of precompound and evaporation contributions.

Fig. 1b. ${ }^{58} \mathrm{Ni}(d, p) E_{d}=56 \mathrm{MeV}$; Proton spectra at $\sim 10^{\circ}$ and $20^{\circ}$. The curves correspond to $\mathrm{EB}+\mathrm{FB}$ calculated results.

Fig. 1 c. ${ }^{27} \mathrm{Al}(\mathrm{d}, \mathrm{p}) \mathrm{E}_{d}=56 \mathrm{MeV}$; Proton spectra at $\sim 10^{\circ}$ and $30^{\circ}$. The curves correspond to $\mathrm{EB}+\mathrm{FB}$ calculated results. 
REFERENCES

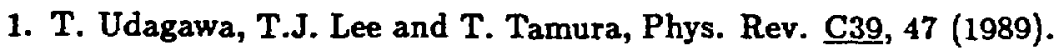

2. G. Bauer, et al., Phys. Rep. 111, 333 (1984).

3. M. Divadeenam, et al., BAPS, $\underline{33} 1581$ (1988).

4. M. Blann and H.K. Vonach, Phys. Rev. C28, 1478 (1983).

5. V.K. Mishra, et al., BAPS, 33,1607 (1988). 


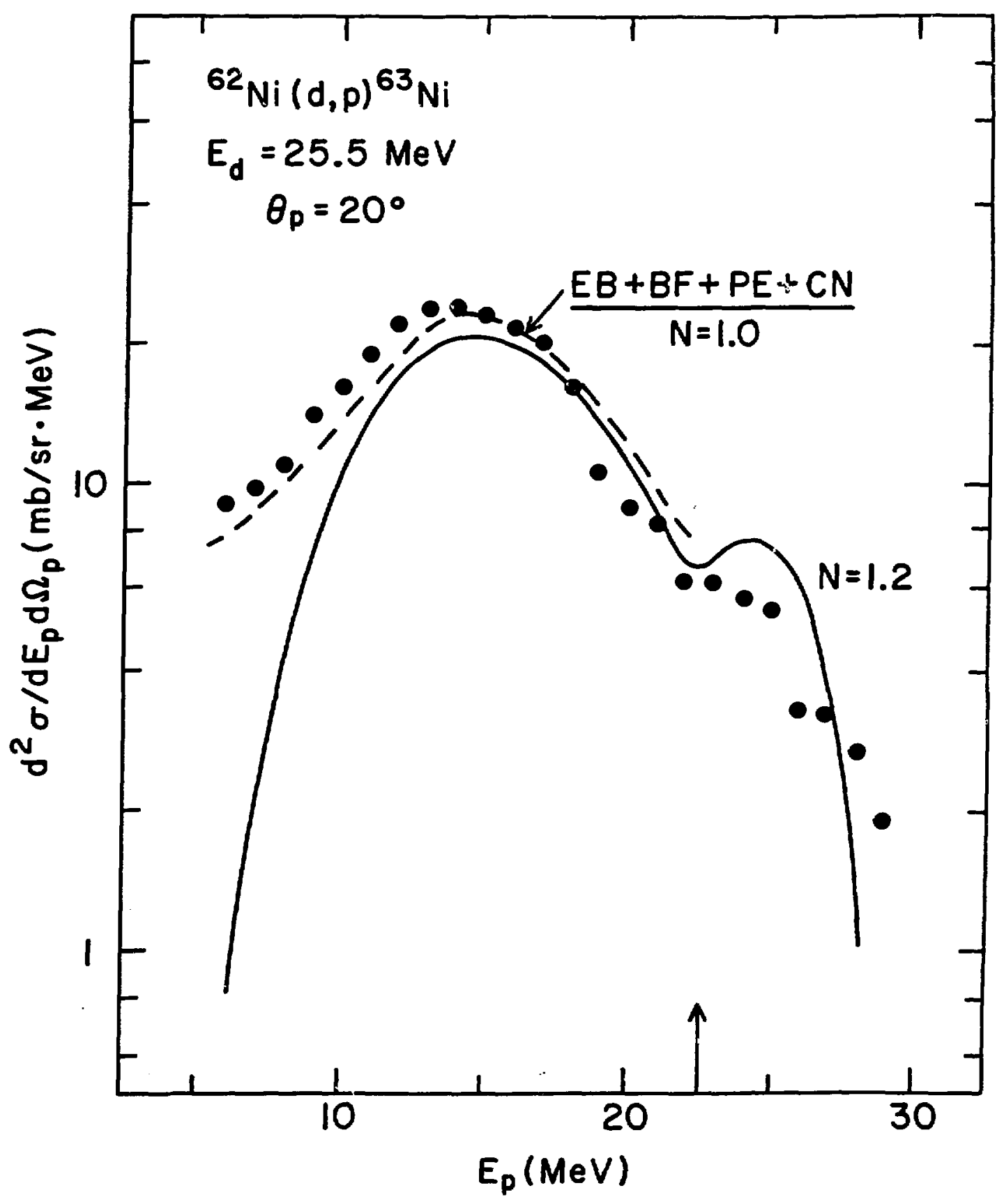




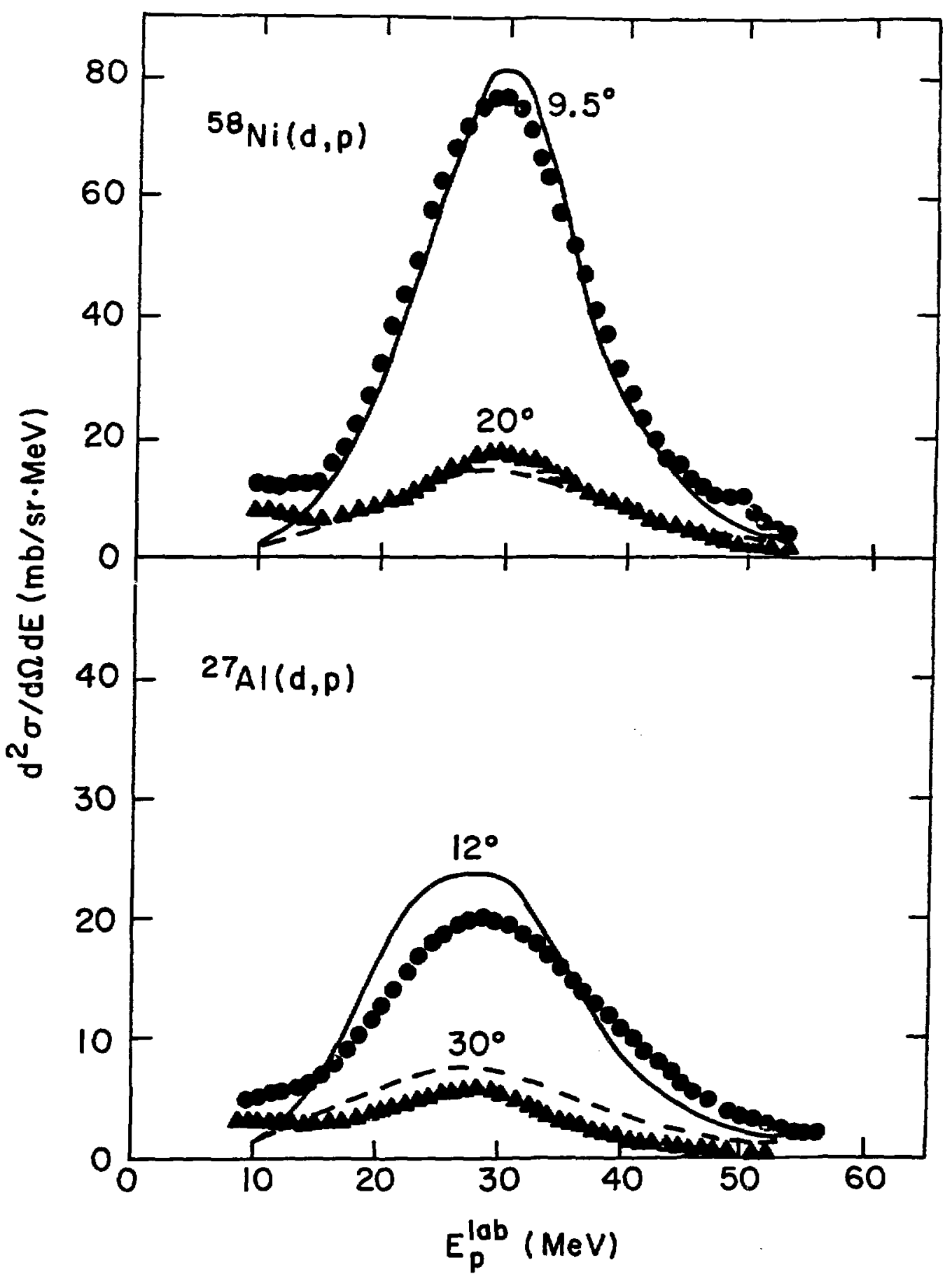

